\title{
Morphology and photoresponse of crystalline antimony film grown on mica by physical vapor deposition
}

\author{
Muhammad Shafa ${ }^{1}$, Zhiming WAnG $^{1,2}$, Muhammad Yasin Naz $^{3,5, *}$, SAdAF Akbar $^{4}$, \\ Muhammad Umar FAROOQ ${ }^{1}$, ABdul GHAFFAR ${ }^{5}$ \\ ${ }^{1}$ Institute of Fundamental and Frontier Sciences, University of Electronics Science and Technology of China, \\ 610054 Chengdu, China \\ ${ }^{2}$ State Key Laboratory of Electronic Thin Films and Integrated Devices, School of Microelectronics and Solid-State \\ Electronics, University of Electronic Science and Technology of China, Chengdu 610054, PR China \\ ${ }^{3}$ Department of Mechanical Engineering, Universiti Teknologi PETRONAS, Bandar Seri Iskandar, \\ 31750 Tronoh, Perak, Malaysia \\ ${ }^{4}$ Zernike Institute for Advanced Materials, University of Groningen, 9747AG Groningen, The Netherlands \\ ${ }^{5}$ Department of Physics, University of Agriculture, 38040-Faisalabad, Pakistan
}

\begin{abstract}
Antimony is a promising material for the fabrication of photodetectors. This study deals with the growth of a photosensitive thin film by the physical vapor deposition (PVD) of antimony onto mica surface in a furnace tube. The geometry of the grown structures was studied via scanning electron microscopy (SEM), X-ray diffraction (XRD), energy-dispersive X-ray spectroscopy (EDX) and elemental diffraction analysis. XRD peaks of the antimony film grown on mica mostly matched with JCPDF Card. The formation of rhombohedral crystal structures in the film was further confirmed by SEM micrographs and chemical composition analysis. The Hall measurements revealed good electrical conductivity of the film with bulk carrier concentration of the order of $10^{22} \Omega \cdot \mathrm{cm}^{-3}$ and mobility of $9.034 \mathrm{~cm}^{2} / \mathrm{Vs}$. The grown film was successfully tested for radiation detection. The photoresponse of the film was evaluated using its current-voltage characteristics. These investigations revealed that the photosensitivity of the antimony film was 20 times higher than that of crystalline germanium.
\end{abstract}

Keywords: vapor solid growth; photodetectors; antimony thin film; rhombohedral mica

(C) Wroclaw University of Technology.

\section{Introduction}

After discovery of the photosensitive thin film, there was a boom in manufacturing of photodetectors due to their potential applications in medical, military and civil industry. The cooled sensors are preferably used in commercial applications of the photosensors [1]. Antimony films grown by vapor deposition on crystalline and non-crystalline substrates have fascinated numerous researchers for their two outstanding properties, firstly as photodetection devices and secondly because of the quantum size effect that occurs when they undergo state transition from amorphous to crystalline state.

*E-mail: yasin603@yahoo.com
A large number of photocathodes and optical filters have already been fabricated from thin films of antimony $[1,2]$. The growth of an antimony thin film on a substrate has appealed to the surface physicists because adsorption of the first layer of antimony on the substrate is nearly unrelaxed while the azimuthal shear between the two antimony atoms is nearly zero. The electrical transport measurements are highly suitable to know the electronic properties of such films; the system becomes quasi-two-dimensional as the film thickness decreases. The transport measurements of crystalline antimony films deposited on insulating substrate have been reported by several authors [3, 4]. The antimony thin films grown on $\mathrm{Si}$ substrate induce quantum size effect due to semimetal-semiconductor transitions [4]. Thin film 
of antimony oxide can be useful for gas sensing materials, electrocatalysts, capacitors, photoconductors and as a constituent of electrochromic devices [5-7].

Guzsvány et al. [3] measured the electrical resistivity of antimony films as a function of film thickness. Hexagonal close-packed phases of antimony deposited by evaporation were revealed by electron microscopy, while the cubic phases were also observed at increased substrate temperatures. Bulk antimony has two known allotropes: the 'black' amorphous form and the 'grey' metallic form with rhombohedral structures. The ability to transitions between crystalline and amorphous phases can be successfully employed in sensing applications [8]. Antimonide materials are attractive for commercial optoelectronic applications where heterojunctions of antimony film with other semiconductor materials allow for nearly complete electromagnetic spectrum [9]. Numerous hot cathodes and optical filters prepared from antimony films are also in daily practice $[8,9]$.

It has been revealed that the growth sequence of an antimony film does not depend much on the vacuum conditions [10]. Antimony is a semimetal with $\mathrm{sp}^{3}$ hybridization whose rhombohedral crystal structures are stable at room temperature [11]. It exhibits 80 times lower plasma frequency than gold, therefore can be potentially used as a host for surface polarization [12]. In addition, the antimony thin films have also been regarded as one of the constituent materials used in electronic devices [13]. The motivation for conducting the research works was primarily an interest in growing the photo-sensitive thin films by the vapor deposition of antimony onto mica surface in a furnace tube. Up to now a physical vapor deposition technique has been used for the structural growth of a uniformly distributed film. The geometry of the grown structures was studied via scanning electron microscopy (SEM), X-ray diffraction (XRD), energy-dispersive X-ray spectroscopy (EDX) and elemental diffraction analysis. The findings of the current investigations are discussed in details in the following sections.

\section{Materials and methods}

The methods for films deposition can be divided into two categories: (i) liquid-based deposition (e.g. chemical solution deposition, electrochemical deposition, Langmuir-Blodgett films and self-assembled monolayers) and (ii) vapor-phase deposition (e.g. evaporation, chemical vapor deposition, molecular beam epitaxy, atomic layer deposition and sputtering) [14-16]. Growth of films on nanoscale involves nucleation which is very vital because it controls the microstructure and crystallinity of the film. There are three basic modes of nucleation: (i) island-layer or Stranski-Krastonov nucleation. (ii) island or Volmer-Weber, and (iii) layer or Frank-van der Merwe as illustrated in Fig. 1. Antimony is a semimetal of rhombohedral structure. It has both metallic and covalent characteristics, therefore the crystallite orientation of the grown films are strongly affected by the evaporation rate.

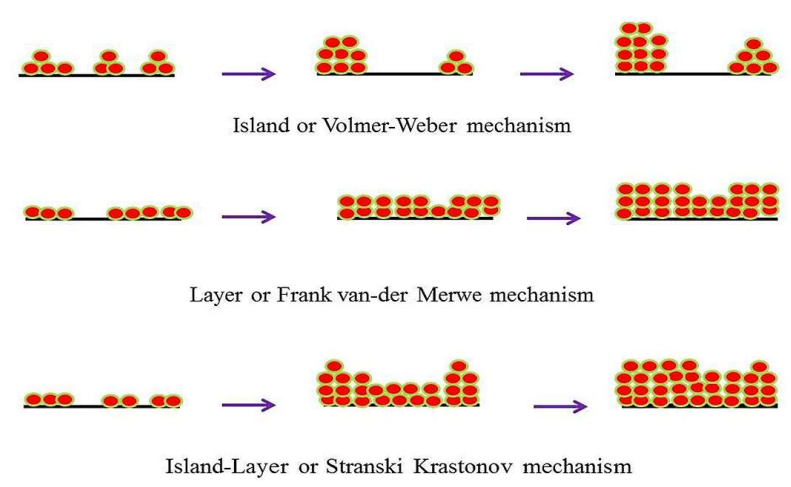

Fig. 1. Illustration of the basic nucleation modes.

Herein, a mica substrate was cleaned with acetone followed by an alcoholic treatment for $5 \mathrm{~min}$ utes. The cleaned substrate was further sonicated using an ultrasonic bath. The $99.99 \%$ pure source metal (polycrystalline antimony) was mechanically meshed and put in a glass tube where it was cleaned via argon bombardment at $873.15^{\circ} \mathrm{C}$. Schematic of the evaporation tube is shown in Fig. 2. This tube was composed of two horizontal heating zones and a quartz tube. The glass boat carrying the metal powder was placed in the central part of the reaction tube, vacuumized up to $0.14 \mathrm{~Pa}$ to ensure 


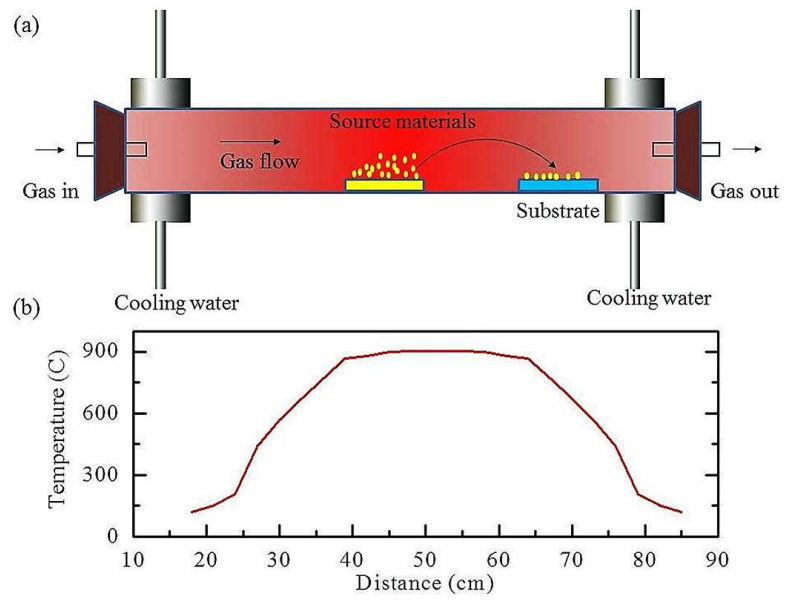

Fig. 2. (a) Schematic of the physical vapor deposition reactor, (b) temperature gradient measured inside the furnace tube.

the complete removal of the residual gases, including oxygen. For the growth of an epitaxial film, the mica substrate was placed in the low temperature zone of the tube at a specific growth temperature of $400{ }^{\circ} \mathrm{C}$ to $500{ }^{\circ} \mathrm{C}$. The source material was vaporized in the high temperature zone of the tube at process temperature of $800{ }^{\circ} \mathrm{C}$ to $900{ }^{\circ} \mathrm{C}$. The temperature ramp rate was fixed to $10{ }^{\circ} \mathrm{C} / \mathrm{min}$. The time for complete vaporization of the source material was assumed to be $1 \mathrm{~h}$. Then the vaporized material was carried by the argon flow to the low temperature zone, where the substrate was placed for epitaxial film growth. The primary nucleation of the film was noted at the growth temperature of $600{ }^{\circ} \mathrm{C}$ to $700{ }^{\circ} \mathrm{C}$, whereas the secondary growth started at the furnace temperature of $900{ }^{\circ} \mathrm{C}$. The details of the growth conditions and vapor concentration are given in Table 1. Once the epitaxial film was completely grown, the furnace tube was set to cool down naturally at room temperature. After $24 \mathrm{~h}$ of cooling, the samples were shifted to an oven operated at $100{ }^{\circ} \mathrm{C}$ to avoid the oxidation. HRXRD, SEM and EDX techniques were used to study the surface morphology, crystallinity and chemical composition of the as grown antimony film [10]. The grown film was further tested for radiation detection. The photoresponse of the film was evaluated using its current-voltage characteristics.

\section{Results and discussion}

\subsection{Temperature gradient}

Before starting the growth process, it was necessary to measure the temperature gradient in the furnace tube. The furnace temperature was raised to $900{ }^{\circ} \mathrm{C}$ and temperature gradient was measured using $100 \mathrm{~cm}$ long wire k-type thermocouple. The temperature measurements were performed by moving the thermocouple inside the tube in steps of $2 \mathrm{~cm}$. The thermocouple was moved to next position in forward direction after each $20 \mathrm{~min}$. The chilled water was circulated through the outer part of the furnace tube to control the jacket temperature. This practice helped us identifying the ideal temperature zone for the antimony film growth. The source and substrate were fixed at the positions found suitable for improved PVD growth of the antimony film. As the tube furnace was $100 \mathrm{~cm}$ long, the furnace temperature changed abruptly by moving from one point to the other one inside the tube. The change in temperature by moving away from the central zone of the tube is plotted in Fig. 2. The temperature plot is composed of three distinct regions. Initially, an abrupt increase in temperature from $200{ }^{\circ} \mathrm{C}$ to $850{ }^{\circ} \mathrm{C}$ is observed in the tube region between $20 \mathrm{~cm}$ and $35 \mathrm{~cm}$. At this point, the temperature reaches almost a constant value and does not vary significantly over a distance between $35 \mathrm{~cm}$ and $65 \mathrm{~cm}$. However, the furnace temperature starts to decline sharply with further increase in distance beyond this point. Therefore the tube region between $35 \mathrm{~cm}$ and $65 \mathrm{~cm}$ was the optimum for PVD growth of the antimony film.

\subsection{XRD study of antimony film}

XRD measurements were performed on asgrown thin film using a Jordan Valley's D1 Evolution equipped with a filter of $\mathrm{CuK} \alpha, \mathrm{K}=1.5406 \AA$ radiation in the reflection mode using glancing angle incidence of $1.5^{\circ}$. A standard $2.2 \mathrm{~kW}$ sealed tube X-ray generator with parabolic multilayer mirror was used for this purpose. Pure mica and mica having antimony film were characterized on the basis of their XRD spectra, as shown in Fig. 3. In the XRD pattern of mica, four major diffraction peaks 
Table 1. Summary of the growth conditions and vapor concentration in furnace tube of PVD reactor (Source temperature $900{ }^{\circ} \mathrm{C}$, growth temperature $441^{\circ} \mathrm{C}$, source before deposition: $0.1044 \mathrm{~g}$, after deposition: $0.000 \mathrm{~g})$.

\begin{tabular}{llllllllllllllll}
\hline Time [min] & 1 & 5 & 8 & 13 & 18 & 23 & 33 & 46 & 66 & 71 & 76 & 81 & 86 & 91 & 160 \\
\hline Temp. $\left[{ }^{\circ} \mathrm{C}\right.$ ] & 300 & 450 & 600 & 770 & 900 & 900 & 900 & 900 & 900 & 900 & 900 & 800 & 700 & 600 & 300 \\
\hline Pressure 0.1 Pa & 1.4 & 1.4 & 1.4 & 1.4 & $\begin{array}{l}1.4 \\
\mathrm{GFC}^{*}\end{array}$ & 1.4 & 1.0 & 1.0 & 8.6 & 8.6 & 7.4 & 7.4 & 7.4 & 8.6 & 1.4
\end{tabular}

${ }^{*}$ GFC: Growth Flow Condition

are seen at $2 \theta$ of $8.7^{\circ}, 17.6^{\circ}, 26.6^{\circ}$ and $45.1^{\circ}$. However, the XRD pattern of mica coated with antimony film shows some additional diffraction peaks at $13.7^{\circ}, 18.1^{\circ}, 27.7^{\circ}$ and $32.0^{\circ}$, which can be assigned to (l 112$),\left(\begin{array}{lll}1 & 0 & 4\end{array}\right),\left(\begin{array}{lll}1 & 0 & 7\end{array}\right)$ and $\left(\begin{array}{lll}0 & 0 & 9\end{array}\right)$ reflections of the textured structure of the rhombohedral antimony indexed as a hexagonal system.

The deposited antimony with a rhombohedral lattice $[17,18]$ revealed good crystallinity of the film. Antimony has a rhombohedral structure with the lattice parameters of $\mathrm{a}=\mathrm{b}=4.3 \AA$, and $\mathrm{c}=$ $11.25 \AA$. The growth of antimony film with polycrystalline texture was also confirmed by SEM and EDX investigations in the following sections. Nevertheless, some of the diffraction peaks, associated with antimony, were missing in the XRD pattern of the coated mica. The absence of these peaks in the diffraction pattern was associated with the smaller particle size of the as-deposited antimony. The particles with the sizes smaller than X-ray coherence length were difficult to trace in the XRD investigations. These results suggest that the thin film grown under the current operating conditions consisted mainly of crystalline rhombohedral antimony [19].

\subsection{EDX and SEM studies of antimony film}

A JSM-6510LV scanning electron microscope was used to study the surface morphology of the antimony film. SEM analysis of the film confirmed that the deposition of antimony on the mica sheet was following an island growth mechanism. The coating was composed of small grains uniformly distributed across the surface of the substrate. There was no change or even variation in the film morphology over the whole surface area of the

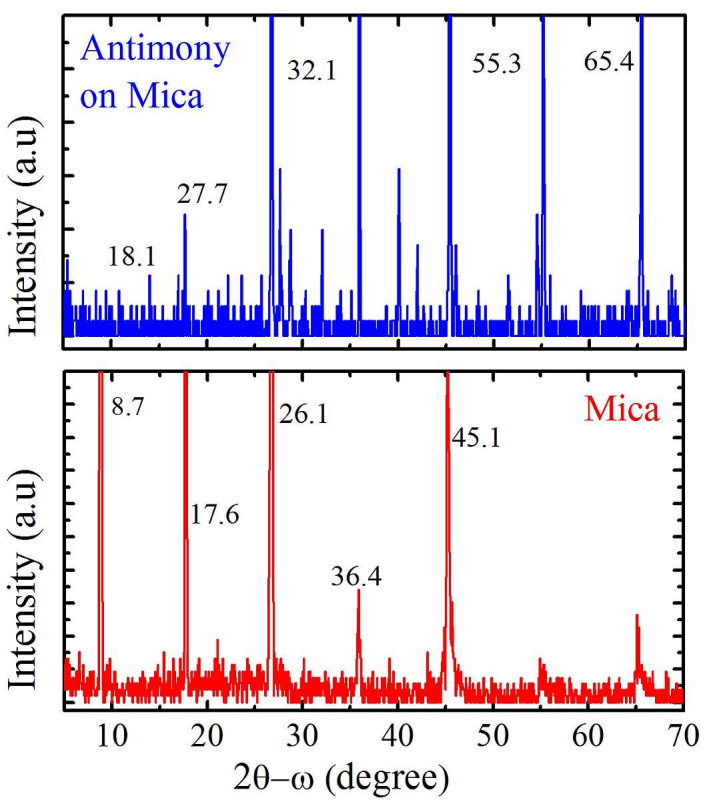

Fig. 3. XRD patterns of antimony coated and uncoated mica.

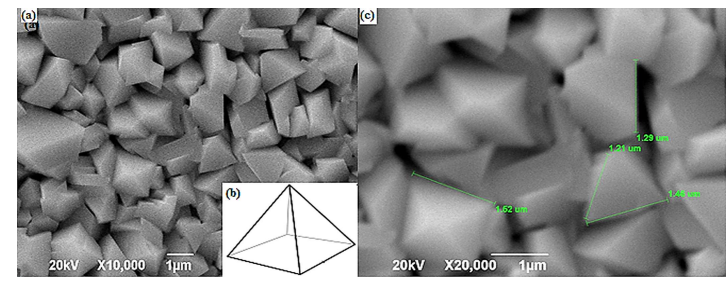

Fig. 4. SEM micrographs of the antimony thin film grown on a mica sheet.

substrate. The uniformly distributed rhombohedral crystalline grains of antimony on the substrate are clearly seen in SEM micrographs shown in Fig. 4. The rhombohedral microstructures with their measured dimensions are also shown in Fig. 4c.

Results of the pre-annealing EDX investigations of the composition of the coated film are 
shown in Fig. 5. The films could be further oxidized due to longer exposure time in open environment or in the annealing treatment where oxygen could react with antimony surface resulting in the formation of oxide film [9]. EDX revealed that the cluster beam was composed of a mixture of crystalline and spherical amorphous particles. The size distribution of the crystalline clusters in SEM images was not consistent with the size estimated from the diffraction patterns [8]. The nanostructures were approximately $1.30 \mu \mathrm{m}$ long and contained only minor amounts of impurities as judged by the EDX. The representative spectra of the preand post-annealed antimony films with rhombohedral structure are shown in Fig. 5 and Fig. 6, respectively. The spectrum in Fig. 5 reveals the presence of small amounts of indium and gold impurities in the antimony films. These impurities were removed after annealing the antimony film at $300{ }^{\circ} \mathrm{C}$ for more than one hour.

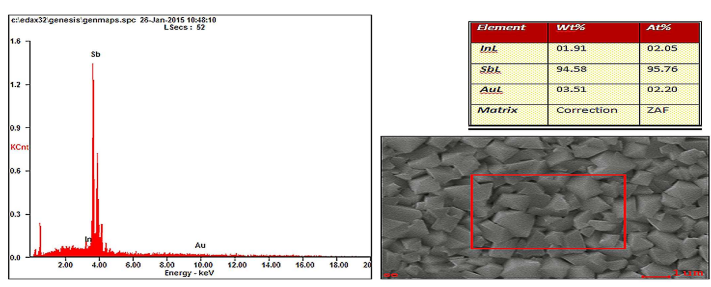

Fig. 5. Pre-annealing EDX and SEM of antimony film.

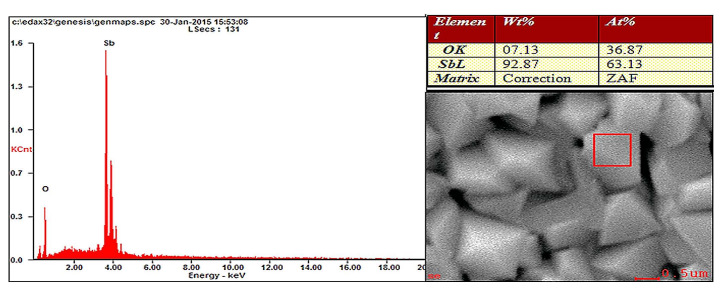

Fig. 6. Post-annealing EDX and SEM of antimony film.

\subsection{Hall measurements and photore- sponse}

Semiconducting behavior of the antimony film was investigated by Hall measurements at room temperature. In these measurements, the carrier concentration was found of the order of $10^{22} \mathrm{~cm}^{-3}$,
Hall mobility of about $9.03 \mathrm{~cm}^{2} / \mathrm{Vs}$ and resistivity of about $10^{-6} \Omega \cdot \mathrm{cm}$. The thin film sensitivity might be due to electron scattering at the surface and grain boundaries. The surface and grain boundary scattering of the electrons significantly contribute to the thin film resistivity. If grain boundary scattering dominates the surface scattering, the temperature dependent part of the resistivity would be identical to that of bulk material. On the other hand, if surface scattering is a dominating factor, the temperature dependent part of the resistivity may deviate from the bulk material resistivity. In the work, the sheet concentration was of the order of $10^{14} \mathrm{~cm}^{-2}$, Hall coefficient of the order of $6.99 \times 10^{-4} \mathrm{~m}^{2} / \mathrm{C}, \Delta \mathrm{R}$ about $5.23 \Omega$ and conductivity of the order of $1.35 \times 10^{4}(\Omega \cdot \mathrm{cm})^{-1}$. These factors primarily depend upon the film thickness while for the higher thicknesses, they seem to be thickness-independent [13]. Such a trend can be attributed to the scattering source associated with the film structure and $\mathrm{V} / \mathrm{H}$ ratio.

In thin film photodetectors, radiation interacts with electrons of the film and changes the electronic energy distribution. The corresponding shift in energy produces an electrical signal which is used to study the photoresponse of the as grown film. The photodetector shows a selective wavelength dependence of the response per unit incident radiation power. The photoresponse of the as grown antimony film on the mica substrate was estimated from its current-voltage characteristics. To the best of our knowledge, nobody has ever successfully measured the photoresponse of a rhombohedral antimony thin film by placing it in light and dark. It was observed that when the sample was placed under visible light there was a notable change in its current-voltage characteristics, as shown in Fig. 7. The current-voltage characteristics exhibited clearly different trends in light and dark scenarios. At the same time, the currentvoltage responses of the same sample under visible light and Xenon lamp showed a good agreement.

\section{Conclusions}

An antimony thin film was deposited on a mica substrate using a simple physical vapor 


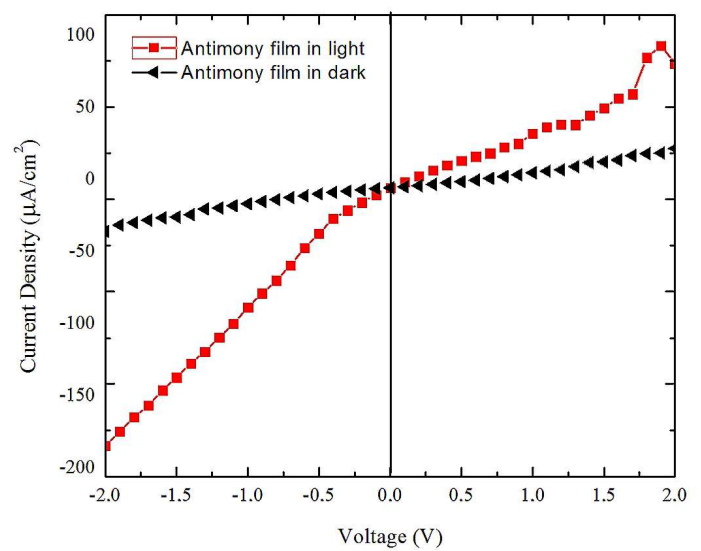

Fig. 7. Current density-voltage (j-V) characteristics of antimony thin film under dark and light scenarios.

deposition method. The surface properties and structural quality of the as grown film were assessed using SEM and XRD techniques. It was concluded that the film was uniformly distributed over the substrate with micro-sized clusters, depending on the growth temperature. XRD studies showed that the antimony thin film had a good rhombohedral crystalline structure, which was further confirmed by SEM micrographs. The Hall measurements showed good electrical conductance of the film with carrier concentration of the order of $10^{22} \mathrm{~cm}^{-3}$ and mobility of $9.034 \mathrm{~cm}^{2} /$ Vs. The as grown antimony film demonstrated high radiation detection ability, which was 20 times higher than that of germanium, as verified by drawing the current-voltage characteristics. The device performance is expected to be improved further by reducing the trap states and surface passivation. The method presented in this paper can be an important alternative to the mid-infrared technology.

\section{Acknowledgements}

The authors acknowledge the financial support from the National Natural Science Foundation of China through the Grant No. NSFC-51272038 and NSFC-61204060.

\section{References}

[1] Hojun R., Cheon S.H., Yang S.W., Yu B.G., Choi C.A., LeE M.L., Kwon S.O., IEEE Sens. J., 2008 (2008), 301.

[2] Mukherjee A., Mitra P., Mater. Sci.-Poland, 33 (2016), 847.

[3] Guzsvány V., Nakajima H., Soh N., Nakano K., Imato T., Anal. Chim. Acta, 658 (2010), 12.

[4] Martinez A., Collazo R., Berrios A.R., Ducoudray G.O., J. Cryst. Growth, 174 (1997), 845.

[5] Binions R., Carmalt C.J., Parkin I.P., Polyhedron, 25 (2006), 3032.

[6] Huković M.M., Babić R., Brinić S., J. Power Sources, 157 (2006), 563.

[7] Pérez O.E.L, SÁnchez M.D., Teijelo M.L., J. Electroanal. Chem., 645 (2010), 143.

[8] Kaufmann M., Wurl A., Partridge J.G., Brown S.A., Eur. Phys. J. D, 34 (2005), 29.

[9] Todd M.A., BAndari G., BAUM T.H., Chem. Mater., 11 (1999), 547.

[10] Ghosh C., Varma B.P., J. Phys. D Appl. Phys., 7 (1974), 5

[11] Wu J.H., Yan J.W., XIE Z.X., Xue Q.K., MaO B.W., J. Phys. Chem. B, 108 (2004), 2773.

[12] Cleary J.W., Medhi G., Shahzad M., Rezadad I., Maukonen D., Peale R.E., BoreMAN G.D., Wentzell S., BuchWald W.R., Opt. Express, 20 (2012), 2693.

[13] Elfalaky A., Appl. Phys. A-Mater., 60 (1995), 87.

[14] NaZ M.Y., Shukrullah S., Ghaffar A., Shakir I., Ullah S., SAgIR M., Surf. Rev. Lett., 21 (2014), 1450056.

[15] Shukrullah S., Mohamed N.M., Shaharun M.S., Diam. Relat. Mater., 58 (2015), 129.

[16] Oyedotun K.O., Ajenifuja E., Olofinjana B., Taleatu B.A., Omotoso E., Eleruja M.A., AJAYi E. O.B., Mater. Sci.-Poland, 33 (2016), 725.

[17] LiU P., Zhong K., Liang C., Yang Q., Tong Y., Li G., Hope G.A., Chem. Mater, 20 (2008), 7532.

[18] Wang Y.W., Hong B.H., LEe J.Y., KiM J.S., KIM G.H., KIM K.S., J. Phys Chem. B, 108 (2004), 16723.

[19] Xue M.Z., Fu Z.W., Electrochem. Commun., 8 (2006), 1250 . 